Received: 14 August 2018

Accepted: 8 November 2018

Published online: 20 December 2018

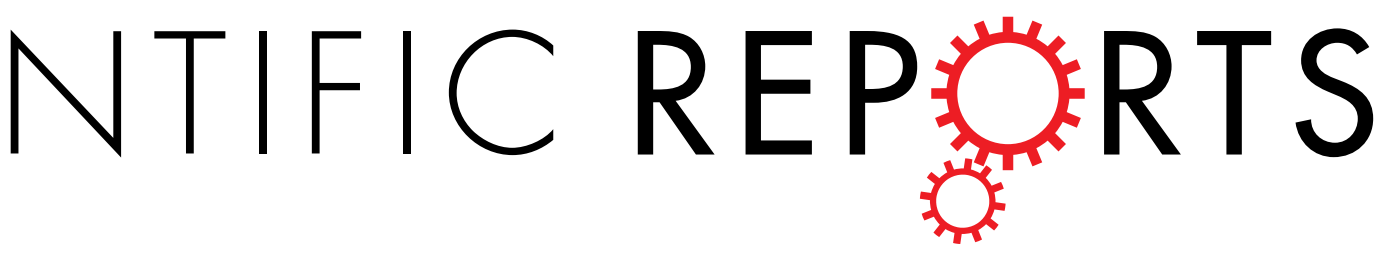

\title{
Integrated analysis of microRNA and mRNA expression profiles in splenomegaly induced by non- cirrhotic portal hypertension in rats
}

Junji Saruwatari ${ }^{1}$, Chao Dong ${ }^{1,2}$, Teruo Utsumi ${ }^{3}$, Masatake Tanaka ${ }^{1}$, Matthew McConnell ${ }^{1}$ \& Yasuko Iwakiri ${ }^{1}$

The spleen plays an important role in the immune and hematopoietic systems. Splenomegaly is a frequent consequence of portal hypertension, but the underlying molecular and cellular mechanisms remain to be fully elucidated. In this study, we have performed a whole-genome microarray analysis combined with histological examination in enlarged spleens isolated from rats with partial portal vein ligation (PPVL) surgery to provide comprehensive profiles of microRNAs and their target mRNAs with a focus on their potential biological functions. A total of 964 mRNAs and 30 microRNAs showed significant differential expression in the spleens of PPVL rats compared to rats undergoing a sham procedure. Twenty-two down-regulated microRNAs were associated with significantly increased genes highly involved in fibrogenic activity and cell proliferation/migration (e.g., Ctgf, Serpine1, Col1a1). Consistently, histological analyses demonstrated increased splenic fibrosis and cell proliferation in the spleens of PPVL rats. Eight up-regulated microRNAs were associated with suppression of genes that are related to interferon-mediated antiviral activity in innate immune responses (e.g., Irf7, Dhx58). In conclusion, we determined a specific microRNA-mRNA network potentially implicated in the tissue fibrosis and cell proliferation in portal hypertension-induced splenomegaly. Our findings provide new insight into the mechanisms for regulation of spleen structure and function.

The spleen is an important organ for the immune system, generating antibodies, removing microbes, and serving as a reservoir for immune cells ${ }^{1,2}$. It also modulates blood components, providing red blood cells when they are deficient during hemorrhagic shock, recycling iron, and capturing circulating platelets ${ }^{1,2}$. Currently, the regulation of spleen structure and function remains to be fully elucidated. Profiling of transcriptomes in the spleen will, therefore, provide novel insights into the regulation of the structure and function.

Splenomegaly is a consequence and an important clinical indicator of portal hypertension ${ }^{3}$. Guidelines addressing portal hypertensive bleeding in cirrhosis recommend routine radiographic assessment of spleen size as an aid to clinical decision-making ${ }^{4}$, and spleen stiffness is increasingly recognized as an important diagnostic tool in the assessment of patients with chronic liver disease ${ }^{5}$. The role of the spleen as active player or passive bystander in portal hypertension, however, remains controversial. Some clinical studies have indicated improvement in portal hypertension following splenectomy in patients ${ }^{6}$. These studies suggest that splenomegaly is an important potential therapeutic target in portal hypertension; however the molecular mechanisms of portal hypertensive splenomegaly remain poorly understood ${ }^{7,8}$. Elucidating these mechanisms may lead to therapeutic options for patients with portal hypertension targeting the spleen that do not carry the substantial risks of surgery.

MicroRNAs (miRNAs) are a class of non-coding RNA molecules that play a key role in a wide range of biological processes, including cell differentiation, proliferation and survival, by binding to complementary target mRNAs, resulting in mRNA translational inhibition or degradation ${ }^{9,10}$. miRNAs have also emerged as critical regulators in the development of immune and inflammatory responses ${ }^{10-12}$. Identifying miRNAs and their target

${ }^{1}$ Section of Digestive Diseases, Yale University School of Medicine, New Haven, CT, USA. ${ }^{2}$ Department of General Surgery, Xiangya Hospital, Central South University, Changsha, China. ${ }^{3}$ VA CT Healthcare System, West Haven, CT, USA. Junji Saruwatari and Chao Dong contributed equally. Correspondence and requests for materials should be addressed toY.I. (email: yasuko.iwakiri@yale.edu) 


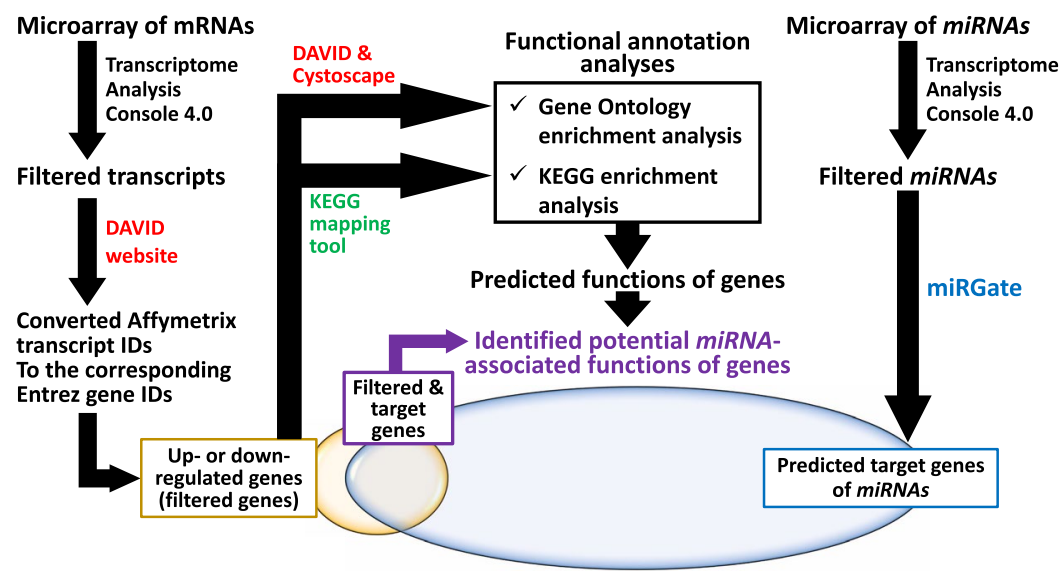

Figure 1. The workflow of mRNA and miRNA data analyses.

mRNAs on a genome-wide scale could thus provide valuable information to understand the mechanisms of splenomegaly and gain insight into its pathological consequences.

The study presented here describes the use of whole-genome microarray analysis combined with histological analysis to provide a comprehensive profile of miRNAs and their target mRNAs linked to pathophysiological changes associated with splenomegaly in rats who have undergone partial portal vein ligation (PPVL) surgery. PPVL, representing non-cirrhotic portal hypertension, is one of the most frequently used experimental models of portal hypertension, allowing us to examine the mechanisms by whcih increased portal venous pressure alone, without other confounding factors, can lead to splenomegaly ${ }^{13}$. The results from the current study reveal a miRNA-mRNA network that defines structural and biological alterations that occur in portal hypertension-induced splenomegaly.

\section{Results}

Transcriptional profile of mRNAs in enlarged spleens of rats with portal hypertension Filtering of microarray data. A flowchart of mRNA and miRNA data analyses for enlarged spleens of rats with portal hypertension and control spleens is presented in Fig. 1. Expression of 1,549 transcripts out of 28,407 genes differed on the microarray between these two groups (Fig. 2). Among them, 964 genes (Supplementary Fig. S1), of which 599 genes were up-regulated and 365 genes were down-regulated in the spleens of PPVL rats, were recorded as protein coding genes in Entrez Gene, the gene-specific database of the National Center for Biotechnology Information ${ }^{14}$. Forty out of the 599 up-regulated genes showed a greater than 1.5 -fold increase with 10 of them increasing by more than 2 -fold, while 41 out of the 365 genes were down-regulated by more than 1.5 -fold with 8 of them decreasing by more than 2 -fold. Table 1 lists the top 15 up- and down-regulated genes in the order of fold-change.

Gene ontology and pathway analyses. To determine biological functions that could be influenced in the spleens of PPVL rats, we performed gene ontology (GO) analysis and categorized the identified genes into biological functions ('GO terms') constructed by biological process, molecular function and cellular component ${ }^{15,16}$. An analysis of all genes up- and down-regulated in the spleens of PPVL rats using the Functional Annotation Chart Tool of the Database for Annotation, Visualization and Integrated Discovery (DAVID) (Fig. 1) identified 335 and $65 \mathrm{GO}$ terms for the genes up- and down-regulated, respectively. GO terms most often annotated were "extracellular space" for the genes up-regulated and "cytoplasm" for the genes down-regulated (Table 2).

Genes up-regulated in the spleens of PPVL rats. Given the results from filtering of microarray data mentioned above, we further charaterized the up-regulated genes associated with the GO term "extracellular space" (Table 2) with identification of additional GO terms to which they were also related. Highly up-regulated genes such as $\alpha$-defensins (RatNP-3b, Np4, Defa5 and Defa11) were also associated with GO terms "defense responses to fungus/bacterium" (Fig. 3A). Genes such as Mmp2 and Serpine1 (also known as PAI-1) were related to a GO term "response to cytokine" (Fig. 3A). Other up-regulated genes related to "extracellular space" were also associated with GO terms such as "cell migration", "positive regulation of cell proliferation", "cellular response to fibroblast growth factor stimulus", "transforming growth factor beta receptor signaling pathway" and "collagen fibril organization" (Fig. 3A).

We then performed the Kyoto Encyclopedia of Genes and Genomes Pathway Database (KEGG)-enrichment analysis with a focus on its three areas, "Cellular Processes", "Environmental Information Processing" and "Organismal Systems", to explore the main pathways in which these up- and down-regulated genes are involved (Fig. 1). First, we determined specific pathways in the "Cellular Processes" area with the genes up-regulated in the spleens of PPVL rats (Fig. 4A). This resulted in identification of two pathways, "focal adhesion" with up-regulated genes such as Col1a1, Col1a2 and Itga8 and "regulation of actin cytoskeleton" with up-regulated genes such as Myh10, Itga8, Fgf1/2 and Pdfgfrb (Fig. 4A, Supplementary Table S1), suggesting these pathways are possibly activated in the spleens of PPVL rats. 


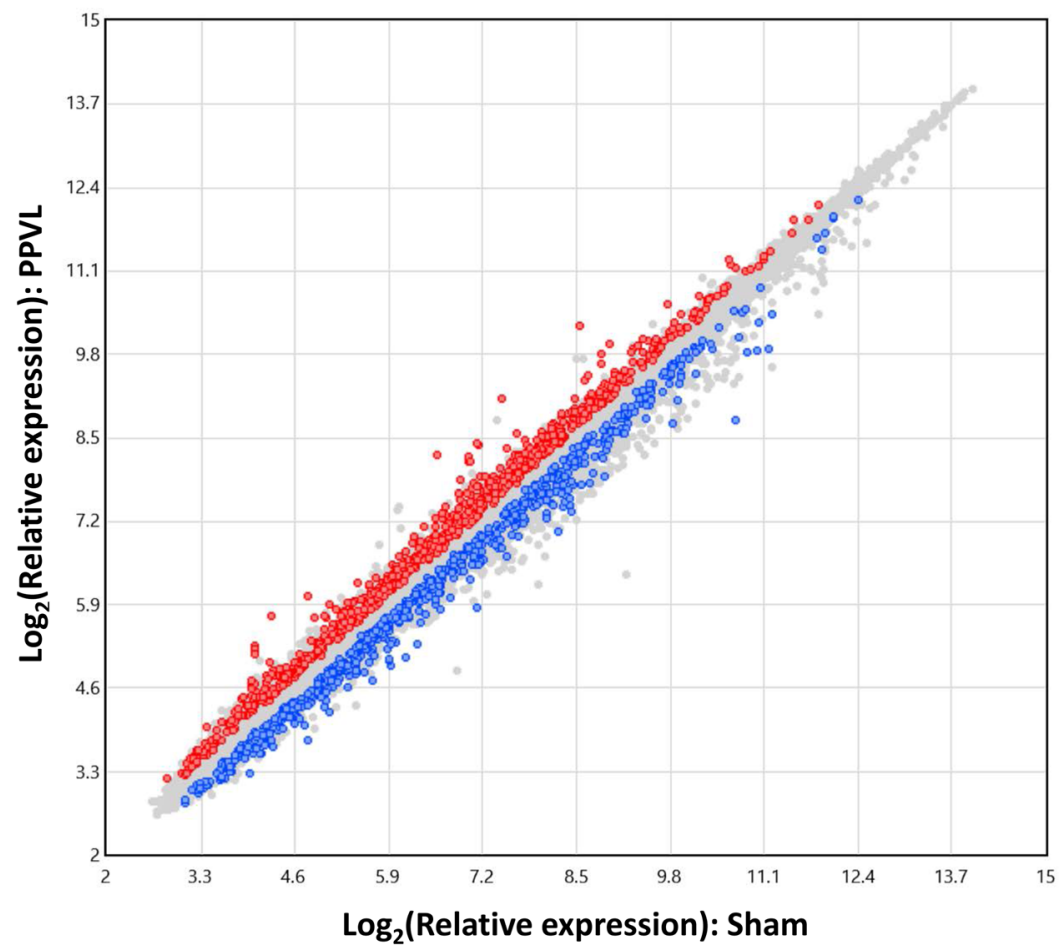

Figure 2. Scatter plot of differentially expressed genes. The plot is color-coded using red for up-regulated genes and blue for down-regulated genes.

Second, we determined pathways in the "Environmental Information Processing" area with the genes up-regulated in the spleens of PPVL rats (Fig. 4B, Supplementary Table S2). The "phosphatidylinositol 3'-kinase (PI3K)-Akt signaling pathway" and "mitogen-activated protein kinase (MAPK) signaling pathway" were identified with the first and second largest numbers of the genes up-regulated in the spleens of PPVL rats (Fig. 4B). However, the main components of the "PI3K-Akt signaling pathway" (i.e., PI3K isoforms and AKT) were not up-regulated (Supplementary Table S2). In the "MAPK signaling pathway", neither the genes of MAPKs (i.e., $E R K, J N K$ and $p 38$ ) nor those of MAPK kinases (i.e., $M E K 1 / 2$ ) were up-regulated in PPVL spleens, while enzymes involved in the deactivation of MAPKs (i.e., Dusp1 and Dusp7) were up-regulated (Supplementary Table S2). The "Hippo signaling pathway" showed the third largest number of the genes up-regulated in PPVL spleens among the pathways in the "Environmental Information Processing" area (Fig. 4B). In the "Hippo signaling pathway", a transcriptional coactivator Wwtr1 (also known as TAZ) and its enhancers Tead1/3 were significantly up-regulated with their target genes, such as Ctgf, Serpine1 and Fgf1, up-regulated as well (Fig. 5, Supplementary Table S2), suggesting that TAZ and TEAD could be involved in controlling organ sizes and/or promoting cell growth and proliferation in PPVL-induced splenomegaly.

In the "Organismal Systems" area, we identified "olfactory transduction", "relaxin signaling pathway" and "axon guidance" with the first, second and third largest numbers of the genes up-regulated in PPVL spleens among the pathways of this classification (Fig. 4C, Supplementary Table S3).

Genes down-regulated in the spleens of PPVL rats. Like the case for the up-regulated genes, given that the genes down-regulated were most commonly annotated to the GO term "cytoplasm" (Table 2), we looked into additional GO terms for the down-regulated genes related to this term. We found that these down-regulated genes were also associated with GO terms "positive regulation of type I interferon production" (including Irf7 and Dhx58), "positive regulation of interferons" (including Irf7 and Dhx58), "innate immune response" and "response to virus" (including Dhx58, Oas1b, Oasl, Oas2, Oasl2, Mx1 and Mx2) (Fig. 3B).

Based on KEGG-enrichment analysis, we identified the "cell cycle", "cellular senescence" and "necroptosis" pathways in the "Cellular Processes" area with the first, second and third largest numbers of the genes down-regulated in PPVL spleens (Fig. 4A, Supplementary Table S1). In the "Environmental Information Processing" area, "cell adhesion molecules (CAMs)", "cytokine-cytokine receptor interaction" and "Jak-STAT signaling pathway" were found with the most substantial numbers of the down-regulated genes, but these pathways were also identified with the genes up-regulated in the spleens of PPVL rats (Fig. 4B, Supplementary Table S2).

In the "Organismal Systems" area, similar to the genes up-regulated, the largest number of the down-regulated genes was annotated to the "olfactory transduction" pathway (Fig. 4C, Supplementary Table S3). The "antigen processing and presentation" (including RT1-T24-3, Ifng and Tfn) and "RIG-I-like receptor signaling pathway" (including Irf7, Isg15 and Ddx58 also known as LGP2), which are implicated in innate immune responses ${ }^{17}$, followed as the second and third pathways in this area (Fig. 4C, Supplementary Table S3). 


\begin{tabular}{|c|c|c|c|c|c|c|}
\hline \multirow[b]{2}{*}{ Entrez Gene ID } & \multirow[b]{2}{*}{ Gene Symbol } & \multirow[b]{2}{*}{ Gene Description } & \multicolumn{2}{|c|}{ Average log2 value } & \multirow[b]{2}{*}{ Fold-change } & \multirow[b]{2}{*}{$P$-value ${ }^{\text {a }}$} \\
\hline & & & Sham & PPVL & & \\
\hline \multicolumn{7}{|c|}{ Up-regulated } \\
\hline 286995 & Defa5 & defensin, alpha 5, Paneth cell-specific & 8.55 & 10.24 & 3.23 & 0.0012 \\
\hline 286958 & Np4 & defensin NP-4 precursor & 6.57 & 8.24 & 3.18 & 0.0016 \\
\hline 498659 & RatNP-3b & defensin RatNP-3 precursor & 7.46 & 9.12 & 3.15 & 0.0009 \\
\hline 613225 & Defa11 & defensin alpha 11 & 4.27 & 5.71 & 2.72 & 0.0172 \\
\hline 314322 & Fos & FBJ osteosarcoma oncogene & 7.11 & 8.41 & 2.45 & 0.0003 \\
\hline 58826 & $\operatorname{Prg} 2$ & proteoglycan 2 & 7.14 & 8.4 & 2.4 & 0.0287 \\
\hline 305941 & Sgcg & sarcoglycan, gamma & 4.77 & 6.02 & 2.38 & 0.0002 \\
\hline 81686 & Mmp2 & matrix metallopeptidase 2 & 7.01 & 8.21 & 2.29 & 0.0101 \\
\hline 29266 & Mcpt2 & mast cell protease 2 & 4.04 & 5.22 & 2.25 & 0.0002 \\
\hline 54289 & Rgs1 & regulator of G-protein signaling 1 & 7 & 8.12 & 2.17 & 0.0061 \\
\hline 29393 & Collal & collagen, type I, alpha 1 & 8.85 & 9.81 & 1.95 & 0.0068 \\
\hline 24617 & Serpine1 & $\begin{array}{l}\text { serpin peptidase inhibitor, clade } \mathrm{E} \text { (nexin, plasminogen } \\
\text { activator inhibitor type 1), member } 1\end{array}$ & 6.9 & 7.85 & 1.93 & $8.10 \mathrm{E}-06$ \\
\hline 25043 & Eln & Elastin & 7.68 & 8.57 & 1.85 & 0.0167 \\
\hline 287382 & Mfap4 & microfibrillar-associated protein 4 & 8.83 & 9.66 & 1.77 & 0.0141 \\
\hline 282836 & Cthrc1 & collagen triple helix repeat containing 1 & 5.08 & 5.9 & 1.76 & 0.0105 \\
\hline \multicolumn{7}{|c|}{ Down-regulated } \\
\hline \begin{tabular}{l|l}
363938 \\
\end{tabular} & Oas2 & $2-5$ oligoadenylate synthetase 2 & 8.34 & 7.5 & -1.78 & 0.001 \\
\hline 361042 & Pck2 & phosphoenolpyruvate carboxykinase 2 (mitochondrial) & 8.41 & 7.55 & -1.81 & 0.0333 \\
\hline 297666 & Klra5 & killer cell lectin-like receptor, subfamily A, member 5 & 5.09 & 4.23 & -1.81 & 0.0109 \\
\hline 309526 & Ifit3 & Interferon-induced protein with tetratricopeptide repeats 3 & 8.31 & 7.43 & -1.84 & $6.26 \mathrm{E}-05$ \\
\hline 295704 & Ube2l6 & ubiquitin-conjugating enzyme E2L 6 & 8.69 & 7.79 & -1.87 & 0.0042 \\
\hline 303538 & Dhx58 & DEXH (Asp-Glu-X-His) box polypeptide 58 & 8.42 & 7.46 & -1.94 & 7.24E-05 \\
\hline 311546 & Tpx2 & TPX2, microtubule-associated & 8.89 & 7.9 & -1.99 & 0.0483 \\
\hline 619560 & Rup2 & urinary protein 2 & 4.79 & 3.78 & -2.01 & 0.0293 \\
\hline 303175 & Hist3h2ba & histone cluster 3, H2ba & 6.3 & 5.28 & -2.03 & 0.0195 \\
\hline 293624 & Irf7 & Interferon regulatory factor 7 & 10.86 & 9.84 & -2.04 & 0.0025 \\
\hline 246268 & Oas1b & $2-5$ oligoadenylate synthetase $1 \mathrm{~B}$ & 8.43 & 7.35 & -2.11 & $5.43 \mathrm{E}-05$ \\
\hline 414788 & RT1-T24-3 & RT1 class I, locus T24, gene 3 & 9.82 & 8.72 & -2.14 & 0.0092 \\
\hline 114247 & Slfn4 & schlafen 4 & 11 & 9.85 & -2.22 & $5.41 \mathrm{E}-05$ \\
\hline 298693 & Isg15 & ISG15 ubiquitin-like modifier & 8.24 & 7.05 & -2.28 & 0.0006 \\
\hline 294090 & Ifitlbl & $\begin{array}{l}\text { interferon-induced protein with tetratricopeptide repeats } \\
\text { 1B-like }\end{array}$ & 10.7 & 8.76 & -3.84 & 0.0167 \\
\hline
\end{tabular}

Table 1. List of top 15 genes up- or down-regulated in the spleens of PPVL rats compared to those of sham rats. ${ }^{a}$ Indicates p-values calculated by an empirical Bayes approach.

Transcriptional profile of miRNAs and their target genes. To examine the potential involvement of miRNAs in differential gene expression in the spleens of PPVL rats, we performed microarray analysis of miRNAs (Fig. 1). One hundred thirty-one miRNAs (with 39 miRNAs up-regulated and 92 miRNAs down-regulated) were differentially expressed out of 1,218 Rattus norvegicus miRNAs (including 728 mature and 490 pre-mature miRNAs) on the microarray. Thirty miRNAs with 22 down-regulated and 8 up-regulated showed more than 1.5 -fold changes (Table 3 ).

We then determined 17,216 genes as predicted target genes of the 22 down-regulated miRNAs using the miRGate database. From these predicted target genes, we extracted 484 genes which were up-regulated in the spleens of PPVL rats (Supplementary Table S4). In other words, most (80.8\%) of the up-regulated genes in the spleens of PPVL rats were predicted as targets of the 22 down-regulated miRNAs. Consistently, genes related to the GO terms determined above were largely predicted target genes (Table 2). They were involved in cell migration and proliferation (Ctgf, Pdgfrb, Fgf1/2, Mmp2, etc.) and cellular response to fibroblast growth factor stimulus/ collagen fibril organization (Serpine1, Eln, Col1a1, Colla2, Col3a1, etc.) (Fig. 6). Likewise, these extracted target genes were associated with KEGG pathways, such as "focal adhesion", "regulation of actin cytoskeleton" and "Hippo signaling pathway" (Supplementary Tables S1, S2 and S3).

Similarly, we determined 13,034 genes as predicted target genes of the eight up-regulated miRNAs and extracted 216 genes which were down-regulated in PPVL spleens (Supplementary Table S5). These extracted genes included genes related to the production of interferons (Irf7 and Dhx58) and innate immune response (Oas1b, Oas2, $M x 1$ and $M x 2$ ) (Fig. 7, Supplementary Tables S1, S2 and S3).

Collectively, these results have demonstrated a close connection between our target gene prediction analysis (miRNAs vs. mRNAs) and our functional annotation analysis (mRNAs vs. GO terms and KEGG pathways), 


\begin{tabular}{|c|c|c|c|c|c|}
\hline \multirow[b]{2}{*}{ GO No. } & \multirow[b]{2}{*}{ GO Term } & \multirow[b]{2}{*}{ Fold Enrichment ${ }^{\mathrm{a}}$} & \multirow[b]{2}{*}{$P$-value ${ }^{b}$} & \multicolumn{2}{|l|}{ Gene } \\
\hline & & & & Fold-change & Gene Name \\
\hline \multicolumn{6}{|l|}{ Up-regulated } \\
\hline \multirow[b]{3}{*}{ GO:0005615 } & \multirow[b]{3}{*}{ extracellular space } & 2.13 & $1.02 \mathrm{E}-10$ & $>2.0$ & Defa5, Defa11, Np4, RatNP-3b, $\underline{\text { Mmp2 }}$ \\
\hline & & & & $>1.5$ & 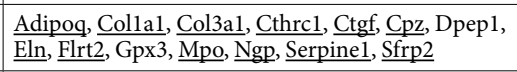 \\
\hline & & & & $>1.0$ & 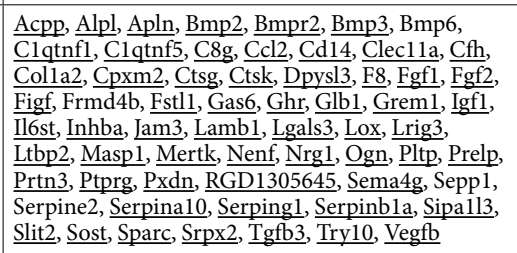 \\
\hline \multicolumn{6}{|c|}{ Down-regulated } \\
\hline \multirow[b]{3}{*}{ GO:0005737 } & \multirow[b]{3}{*}{ cytoplasm } & 1.20 & 0.02481 & $>2.0$ & $\underline{\text { Irf7 }}$ \\
\hline & & & & $>1.5$ & 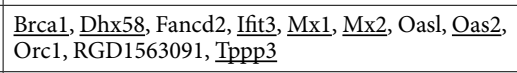 \\
\hline & & & & $>1.0$ & 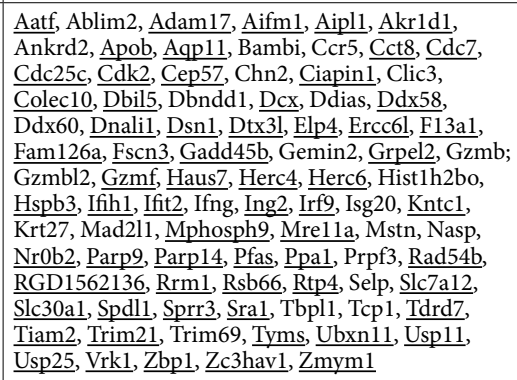 \\
\hline
\end{tabular}

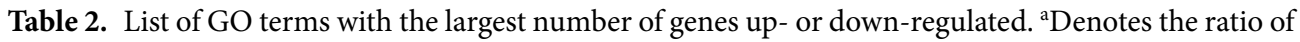
the number of genes in the gene set to the expected number in the category based on the human database. bIndicates p-values adjusted by the multiple test Benjamini adjustment. The underlined genes are predicted targets for up- or down-regulated miRNAs in the spleens of PPVL rats.

providing an integrated network of miRNAs and mRNAs relevant to the pathophysiology of the spleen observed in PPVL rats.

A significant increase in fibrosis and cell proliferation in the spleens of PPVL rats. Given that a substantial number of the up-regulated genes, most of which are potential targets of the down-regulated miRNAs, were associated with cell proliferation/migration and pro-fibrogenic signaling (Figs 3 and 6), we examined the levels of splenic fibrosis and cell proliferation in our model. Consistent with transcriptional profiles of mRNAs and miRNAs, PPVL rats demonstrated significantly increased spleen weights compared to sham rats (Fig. 8A). Splenomegaly in PPVL rats was associated with significantly increased fibrosis as indicated by Sirius red staining (Fig. 8B). In addition, significantly increased cell proliferation was observed in the spleens of PPVL rats, compared to those of sham rats (Fig. 8C). These observations of increased fibrosis and cell proliferation in enlarged spleens of PPVL rats verify the changes in profiles of miRNAs and corresponding mRNAs found in this study.

\section{Discussion}

This is the first study to determine the integrated miRNA-mRNA network in splenomegaly and its potential roles in the underlying molecular mechanisms and biological changes. The current study has identified unique transcriptome profiles regulating biological processes in splenomegaly induced by PPVL, a model of non-cirrhotic portal hypertension. This study has determined 22 down-regulated miRNAs showing more than 1.5 -fold change in the spleens of PPVL rats (Table 3), which included miRNAs that have been implicated in the promotion of fibrosis, cell proliferation and migration (e.g., $m i R-141-3 p)^{18-20}$ and the regulation of inflammatory responses (e.g., let-7i, $m i R-146 b)^{10,11}$. Further, our target prediction analysis has identified that among predicted target genes of these 22 down-regulated miRNAs, genes known to be highly involved in fibrogenic activity, cell proliferation and migration (e.g., Colla1, Serpine1, Ctgf) were up-regulated in the spleens of PPVL rats (Fig. 6). Importantly, in agreement with these transcriptome profiles, histological analyses have demonstrated increased splenic fibrosis and cell proliferation (Fig. 8). Collectively, our study has identified miRNAs and their target mRNAs, which are potentially involved in splenic fibrosis and cell proliferation observed in PPVL-induced splenomegaly.

This study has also determined 8 up-regulated miRNAs in the spleens of PPVL rats (Table 3), which included miRNAs that have been implicated in the immune response (e.g., $m i R-184, m i R-125)^{21,22}$. Among predicted target genes of these up-regulated miRNAs, genes related to IFN- $\alpha$ and $\beta$ production as well as the innate immune response (e.g., Irf7, Dhx58, Oas1b, Mx2) were down-regulated in PPVL-induced splenomegaly (Fig. 7). In contrast, genes encoding several $\alpha$-defensins (RatNP-3b, Np4, Defa5 and Defa11) and Prg2, which have potential implications in antimicrobial and antiviral activities in vivo ${ }^{23-25}$, were significantly up-regulated (Fig. 3B), but except for Prg2, were not associated with the miRNAs down-regulated in the spleens of PPVL rats 
(Supplementary Table S4). These findings may indicate potential distinctive alterations in defense mechanisms in PPVL-induced splenomegaly with miRNA-dependent interferon-mediated antiviral activities being deactivated, but with miRNA-independent defense response by $\alpha$-defensins activated. Regarding $\alpha$-defensins, other subtypes, $\alpha$-defensin-1 and -2, are also known to induce fibroblast proliferation and fibrosis in liver and lung tissues ${ }^{26-28}$. Utilizing the strength of our unbiased screening approach to discover unexpected connections in biological systems, we have identified an association between up-regulated $\alpha$-defensins in the spleen and splenic fibrosis. Given the role of $\alpha$-defensins in fibrosis in other organ systems, this suggests a novel mechanism of portal hypertensive splenic fibrosis warranting further investigation.

Besides portal hypertension, many other pathological conditions such as infection and hematological malignancies can cause splenomegaly ${ }^{2,13}$. Gene expression profiles of splenomegaly may differ according to etiology. Schistosoma infection is a frequent cause of portal hypertension and subsequent splenomegaly in developing countries $^{29}$. A whole-genome microarray analysis was performed recently in enlarged spleens of mice infected with Schistosoma japonicum ${ }^{30}$. In comparison with our results, at least three distinct gene expression profiles are noted. First, extracellular matrix (ECM)-related gene expression (e.g., Ctgf and Fgf1) was significantly decreased in the spleens of Schistosoma-infected mice ${ }^{30}$, whereas these genes were significantly up-regulated in our study (Table 2, Fig. 3A). Second, in contrast with our study (Table 2, Fig. 3B), Schistosoma-induced splenomegaly was related to up-regulation of several interferon-inducible genes such as Irf7, Oas2 and Ifit2. Third, Schistosoma-induced splenomegaly showed decreased expression of lymphocyte chemokines (e.g., Cxcl13, Ccl19 and $C c l 21)^{30}$, while no changes were observed in our study. These differences in gene expression profiles may indicate different mechanisms underlying splenomegaly according to etiology.

Other studies also showed that splenomegaly was associated with splenic fibrosis in rats with PPVL ${ }^{31}$ and that mTOR signaling promoted splenomegaly in these rats ${ }^{31,32}$. While splenic fibrosis was in agreement with our study, our results do not implicate genes related to mTOR signaling in splenomegaly. In these previous studies, expression of phosphorylated 4E-BP1 and p70-S6K proteins, two direct downstream targets of the mTOR kinase, were used to measure the activity of $\mathrm{mTOR}^{31,32}$. The discrepancy between our results and these previous findings may be due to differences between the transcriptional regulation we determined and the posttranslational regulation of downstream mTOR signaling (i.e., phosphorylated states of 4E-BP1 and p70-S6K) ${ }^{31,32}$.

Increased ECM levels may promote splenomegaly, since mechanical signals from the ECM (e.g., stiffness and elasticity) may help splenic cells to proliferate, differentiate and prevent cell death ${ }^{33}$. Mechanical signals induce activation of YAP/TAZ transcriptional coactivators, both of which are downstream transducers of the Hippo signaling pathway and essential effectors of ECM mechanical cues ${ }^{34}$. YAP/TAZ activity is key to the growth of organs, amplification of tissue-specific progenitor cells during tissue renewal and regeneration, and cell proliferation $^{34}$. KEGG enrichment analysis of this study identified a substantial number of up-regulated genes that are involved in the Hippo signaling pathway, including TAZ, TEAD (a key DNA-binding platform for YAP/TAZ) and their downstream genes such as Ctgf, Serpine1 and Fgf1 (Fig. 5, Supplementary Table S2). These downstream genes are known to play substantial roles in many cellular responses and pathological processes including cell adhesion, proliferation, migration, and tissue fibrosis ${ }^{34-37}$. Further studies linking the Hippo signaling pathway and YAP/TAZ activation to increased splenic fibrosis and cell proliferation may provide a novel mechanistic insight into PPVL-induced splenomegaly.

One previous study using microarray determined gene expression profiles in blood samples from patients with idiopathic portal hypertension ${ }^{38}$. The study showed up-regulation of genes that are involved in the actin cytoskeleton, actin binding and cytoskeletal protein binding ${ }^{38}$ as well as down-regulation of genes primarily related to the immune system, suggesting immunological abnormalities in idiopathic portal hypertension ${ }^{38}$. Similarly, our functional annotation analyses indicated increased actin cytoskeleton (Fig. 4A) and immunological abnormalities (Fig. 3) in the spleens of PPVL rats. These similarities in gene expression profiles may suggest that mRNAs identified in human blood samples are potentially derived from the spleen.

Another study performed microarray analysis of miRNAs in splenic macrophages associated with hypersplenism due to portal hypertension in patients with hepatitis B virus (HBV)-related cirrhosis, and identified 99 differentially expressed miRNAs in splenic macrophages of portal hypertensive patients ${ }^{39}$. Those miRNAs included $h s a-m i R-184$ and $h s a-l e t-7 i$. We found that their corresponding rat miRNAs were also differentially expressed in the spleens of PPVL rats (Table 3 ) and were associated with mRNAs involved in cell proliferation and innate immune responses (Figs 6 and 7, Supplementary Tables S4 and S5). In these human splenic macrophages, only one miRNA, has-miR-615-3p, was significantly up-regulated ${ }^{39}$. has-miR-615-3p represses expression of ligand-dependent nuclear receptor corepressor, which is a derepressor of peroxisome proliferator-activated receptor gamma ${ }^{40}$. In rats, no miRNA corresponding to $h s a-m i R-615-3 p$ has been identified in the database at miRbase (http://www.mirbase.org/). Therefore, this study was not able to verify differential expression of $m i R-615-3 p$ in the spleens of PPVL rats.

Another interesting observation in our study is that a considerable number of olfactory receptor genes involved in the "olfactory transduction" pathway were up- and down-regulated in the spleens of PPVL rats (Fig. 4C, Supplementary Table S3). Given that olfactory receptors may play a role in non-chemosensory tissues ${ }^{41}$ and that these receptors have been found outside the olfactory system including the spleen ${ }^{42,43}$, an identification of their ligands and signal transduction in the spleen may provide a new insight into the functional significance of olfactory receptors.

Lastly, of note is the recent direction of developing computational models for prediction of disease-associated miRNAs. Computational models are increasingly considered as an important means for identification of novel miRNA-disease associations and are expected to significantly reduce the time and cost of biological experiments ${ }^{44}$. For example, Chen et al. recently proposed several computational models for miRNA-disease associations ${ }^{45-48}$. One of them is the Bipartite Network Projection for miRNA-Disease Association prediction (BNPMDA) model based on the rating-integrated bipartite network recommendation and known miRNA-disease associations ${ }^{46}$. 
A

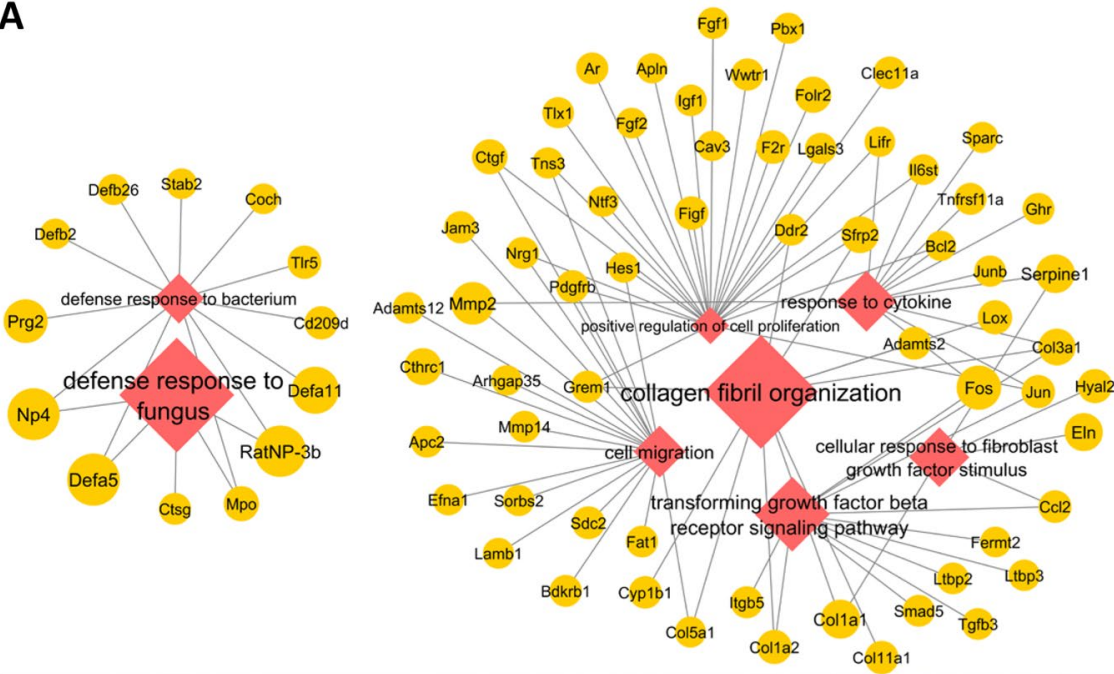

B

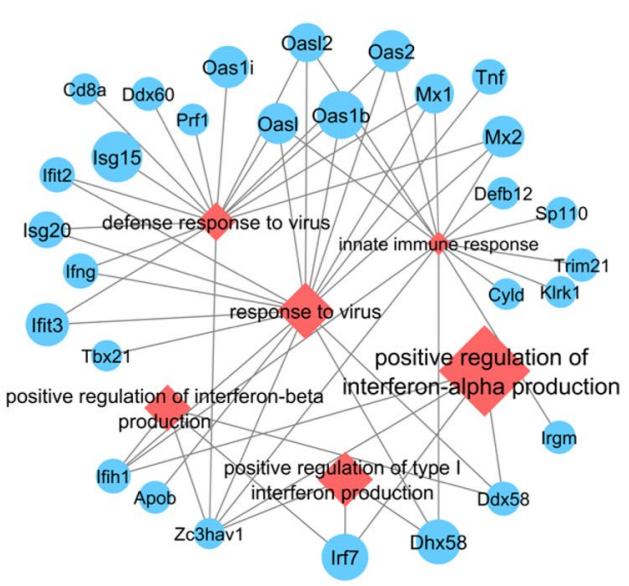

Figure 3. GO terms and their related genes up- or down-regulated in the spleens of PPVL rats. (a) The network consists of GO terms (red diamonds) and their related up-regulated genes (yellow circles). (b) The network consists of GO terms (red diamonds) and their related down-regulated genes (blue circles). The sizes of circles and diamonds represent the fold-change and the fold-enrichment, respectively, in the spleens of PPVL rats compared to those of sham rats.

In this model, using agglomerative hierarchical clustering, they constructed bias ratings between miRNAs and diseases based on miRNA functional similarities and disease semantic similarities. With confirmation of its effectiveness by several validation analyses, they concluded that the BNPMDA model could be an effective computational model for prediction of potential miRNA-disease associations at a high accuracy level.

As also shown in the BNPMDA model, the development of computational models rests on experimentally verified miRNA-disease associations ${ }^{44}$. Currently, however, data volumes of miRNA-disease associations identified by experimental methods are limited. In addition, because there is no information available regarding the miRNA-associated regulation of mRNA expression in portal hypertension-induced splenomegaly, this study provides useful information for further prediction of miRNAs related to the pathophysiology of the spleen in portal hypertension as well as non-cirrhotic portal hypertension-related miRNAs, and thus contributes to unmet needs for accumulation of data on miRNA-disease associations.

In conclusion, we have identified a wide range of gene expression profiles, leading to unique biological consequences, such as splenic fibrosis and cell proliferation as well as potential alterations in immune responses, in PPVL-induced splenomegaly. The novel pathways discovered in this study provide the foundation for future targeted investigation into the mechanisms of splenomegaly in portal hypertension.

\section{Methods}

Animal experiments. All animal experiments were approved by the Institutional Animal Care and Use Committees of Yale University and the Veterans Affairs Connecticut Healthcare System and were performed in accordance with the National Institutes of Health Guide for the Care and Use of Laboratory Animals.

A total of six male Sprague-Dawley rats weighing 300-350 g were used. PPVL or sham surgery was performed in 3 rats each, as described previously ${ }^{49}$. In brief, after midline abdominal incision under anesthesia, the portal 
A

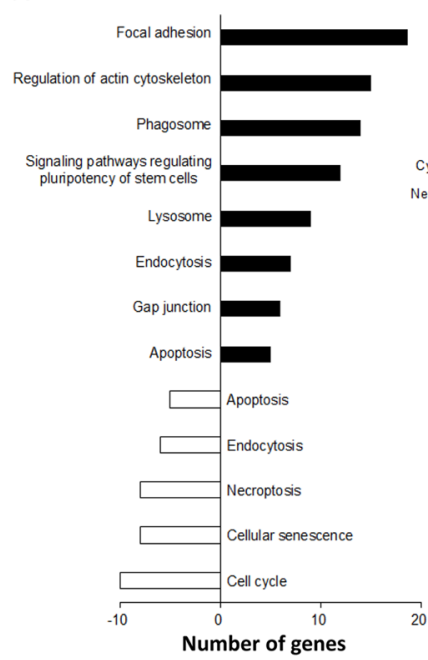

B

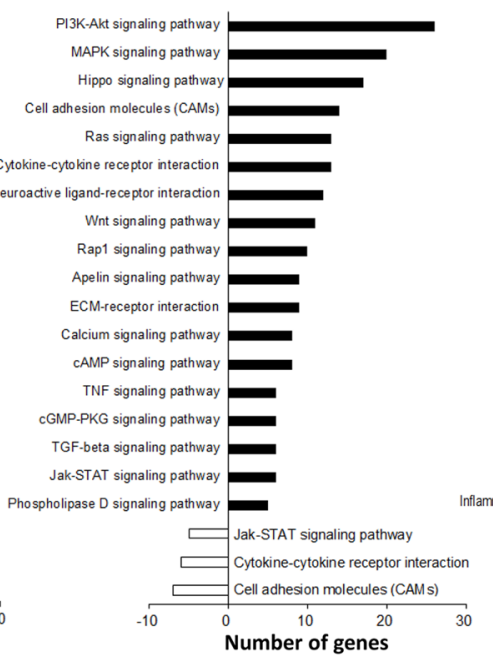

C

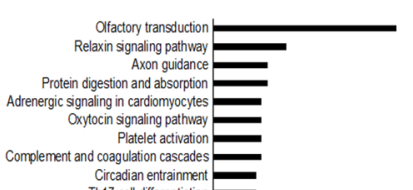

曰 Number of genes up-regulated $\square$ Number of genes down-regulated
(positive values)

Figure 4. The KEGG pathways with more than five genes up- or down-regulated in three pathway areas: (A) "Cellular Processes", (B) "Environmental Information Processing" and (C) "Organismal Systems". The positive and negative numbers represent the number of genes up- and down-regulated, respectively, in the spleens of PPVL rats.

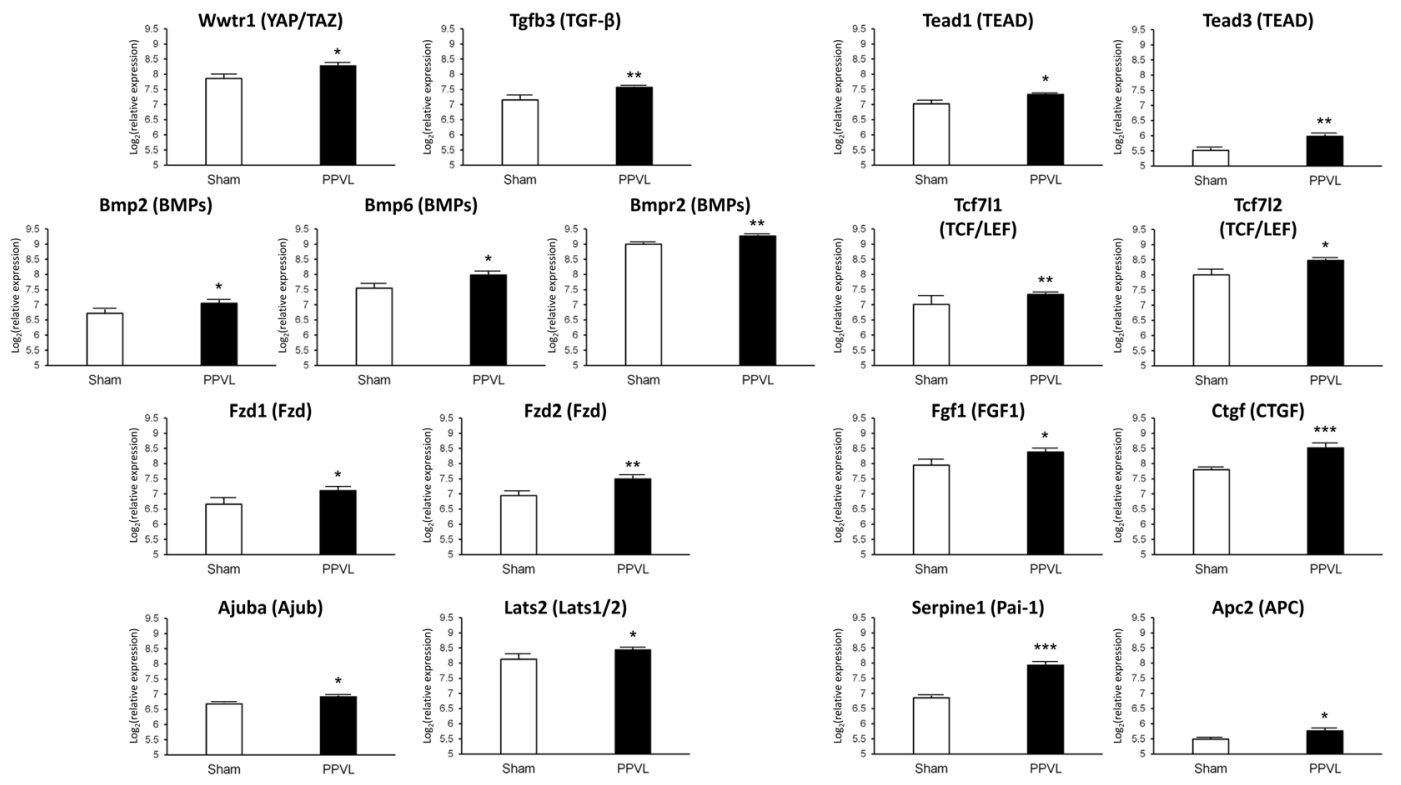

Figure 5. The mean relative expression of genes involved in the KEGG "Hippo signaling pathway". The letters in parenthesis represent the terms cited in the "Hippo signaling pathway" on the KEGG website (http://www. genome.jp/kegg-bin/show_pathway?map04390). “*”, “**” and “***” indicate significance at $\mathrm{p}<0.05, \mathrm{p}<0.01$ and $\mathrm{p}<0.001$, respectively, compared to sham group.

vein was separated from surrounding tissue. A 3-0 silk suture was tied around the portal vein and a 20-gauge blunt-end needle lying along it. Subsequent removal of the needle yielded a calibrated stenosis of the portal vein. In sham-operated rats, the same operation was performed, but no suture was placed. Spleens were collected ten days after surgery when portal hypertension and splenomegaly were fully established in rats with PPVL.

Histological assessment. Formalin-fixed and paraffin-embedded spleen tissue blocks were cut into $5-\mu \mathrm{m}$-thick sections. Sections were stained with hematoxylin and eosin (H\&E) solution for histopathological analysis and also with Sirius red to assess splenic collagen content. These histological assessments were conducted according to the methods we previously reported ${ }^{50,51}$. Immunostaining was performed for Ki67 (Abcam, Cambridge, MA) to determine proliferating cells and alpha-smooth muscle actin ( $\alpha$-SMA) (a marker of myofibroblasts and fibroblastic reticular cells) (Dako, Carpinteria, CA). Sections were visualized by light microscopy 


\begin{tabular}{|c|c|c|c|c|}
\hline \multirow[b]{2}{*}{ microRNA } & \multicolumn{2}{|c|}{ Average $\log 2$ value } & \multirow[b]{2}{*}{ Fold-change } & \multirow[b]{2}{*}{$P$-value } \\
\hline & Sham & PPVL & & \\
\hline \multicolumn{5}{|l|}{ Down-regulated } \\
\hline rno-miR-3584-3p & 3.14 & 1.44 & -3.26 & 0.0115 \\
\hline rno-miR-141-3p & 5.26 & 3.69 & -2.97 & 0.0284 \\
\hline rno-let-7i-3p & 5.53 & 4.57 & -1.94 & 0.0151 \\
\hline rno-miR-223-5p & 2.58 & 1.63 & -1.93 & 0.0195 \\
\hline rno-miR-219b & 2.66 & 1.71 & -1.93 & 0.0128 \\
\hline rno-miR-6318 & 4.01 & 3.06 & -1.92 & 0.0106 \\
\hline rno-miR-147 & 7.09 & 6.22 & -1.83 & 0.0814 \\
\hline rno-miR-3564 & 2.48 & 1.65 & -1.78 & 0.0184 \\
\hline rno-miR-34b-3p & 6.13 & 5.31 & -1.76 & 0.0183 \\
\hline rno-miR-664-1-5p & 7.16 & 6.35 & -1.75 & 0.0273 \\
\hline rno-miR-146b-3p & 2.77 & 2.00 & -1.71 & 0.01 \\
\hline rno-miR-1949 & 9.27 & 8.51 & -1.69 & 0.0627 \\
\hline rno-miR-1224 & 9.56 & 8.82 & -1.67 & 0.0042 \\
\hline rno-miR-18a-5p & 11.26 & 10.53 & -1.66 & 0.08 \\
\hline rno-miR-582-3p & 4.36 & 3.63 & -1.66 & 0.0041 \\
\hline rno-miR-551b-3p & 2.26 & 1.53 & -1.66 & 0.0452 \\
\hline rno-miR-20b-3p & 6.06 & 5.35 & -1.64 & 0.0514 \\
\hline rno-miR-130b-3p & 10.86 & 10.25 & -1.53 & 0.0702 \\
\hline rno-miR-1188-3p & 2.65 & 2.04 & -1.53 & 0.0693 \\
\hline rno-miR-770-3p & 3.79 & 3.17 & -1.53 & 0.0255 \\
\hline rno-miR-409a-3p & 6.05 & 5.45 & -1.52 & 0.0481 \\
\hline rno-miR-874-5p & 3.07 & 2.48 & -1.51 & 0.0329 \\
\hline \multicolumn{5}{|l|}{ Up-regulated } \\
\hline rno-miR-541-5p & 6.94 & 7.56 & 1.53 & 0.007 \\
\hline rno-miR-193-5p & 6.49 & 7.13 & 1.56 & 0.0553 \\
\hline rno-miR-125b-1-3p & 5.07 & 5.77 & 1.63 & 0.0287 \\
\hline rno-miR-411-5p & 4.66 & 5.4 & 1.67 & 0.0388 \\
\hline rno-miR-351-3p & 4.55 & 5.3 & 1.67 & 0.0386 \\
\hline rno-miR-31a-5p & 8.02 & 8.97 & 1.93 & 0.0635 \\
\hline rno-miR-184 & 5.2 & 6.32 & 2.17 & 0.0755 \\
\hline rno-miR-31a-3p & 1.98 & 3.79 & 3.51 & 0.0344 \\
\hline
\end{tabular}

Table 3. List of microRNAs down- or up-regulated more than 1.5-fold in the spleens of PPVL rats compared to those of sham rats. ${ }^{\mathrm{a}}$ Indicates p-values calculated by an empirical Bayes approach.

and images were acquired using an Olympus camera (Olympus, Japan) and Zeiss fluorescence microscopy (Zeiss, Germany). Analysis of digitized images was performed using ImageJ and Prism software.

Isolation of total RNA including miRNA from spleen specimens. Total RNA including miRNA was isolated from approximately $50 \mathrm{mg}$ of frozen whole spleens of PPVL and sham rats using the TRIzol reagent (Thermo Fisher Scientific, Waltham, MA) according to the manufacturer's instructions. Purified RNA quantity and quality were determined by measuring the ratio of absorbance at $260 \mathrm{~nm}$ to that at $280 \mathrm{~nm}$ (A260/A280) using a NanoDrop 2000 Spectrophotometer (Thermo Fisher Scientific) and by on-chip capillary electrophoresis using Bioanalyzer 2100 (Agilent Technologies, Santa Clara, CA). We used the samples with the A260/A280 ratio of $>1.9$ and with the RNA integrity number of $>9$ for microarray analyses.

Microarray hybridization. cRNA synthesis and whole genome microarray analysis were carried out at the Yale Center for Genomic Analysis (Yale University). Single-stranded cDNA was synthesized by reverse transcription using poly(A) RNA present in starting total RNA samples. Single-stranded cDNA was then converted into double-stranded cDNA and purified using Affymetrix Sample Cleanup Module (Thermo Fisher Scientific). In vitro transcription reaction was then carried out overnight in the presence of biotinylated UTP and CTP to produce biotin-labeled cRNA from the double-stranded cDNA. The resulting cRNA was fragmented in the presence of heat and $\mathrm{Mg}^{2+}$ before hybridization to the test array. Microarray analyses of mRNAs and miRNAs were performed using Affymetrix GeneChip ${ }^{\circledR}$ Rat Gene 2.0 ST Array (Thermo Fisher Scientific) and GeneChip ${ }^{\circledR}$ miRNA 4.0 Array (Thermo Fisher Scientific) in conjugation with the FlashTag ${ }^{\circledR}$ Biotin HSR RNA Labeling Kit (Thermo Fisher Scientific), respectively. All reactions and hybridizations were carried out according to the manufacturer's protocol. These arrays were washed with the GeneChip ${ }^{\circledR}$ Fluidics Station 450 (Thermo Fisher Scientific) and scanned with the GeneChip ${ }^{\circledR}$ Scanner 3000 (Thermo Fisher Scientific). 


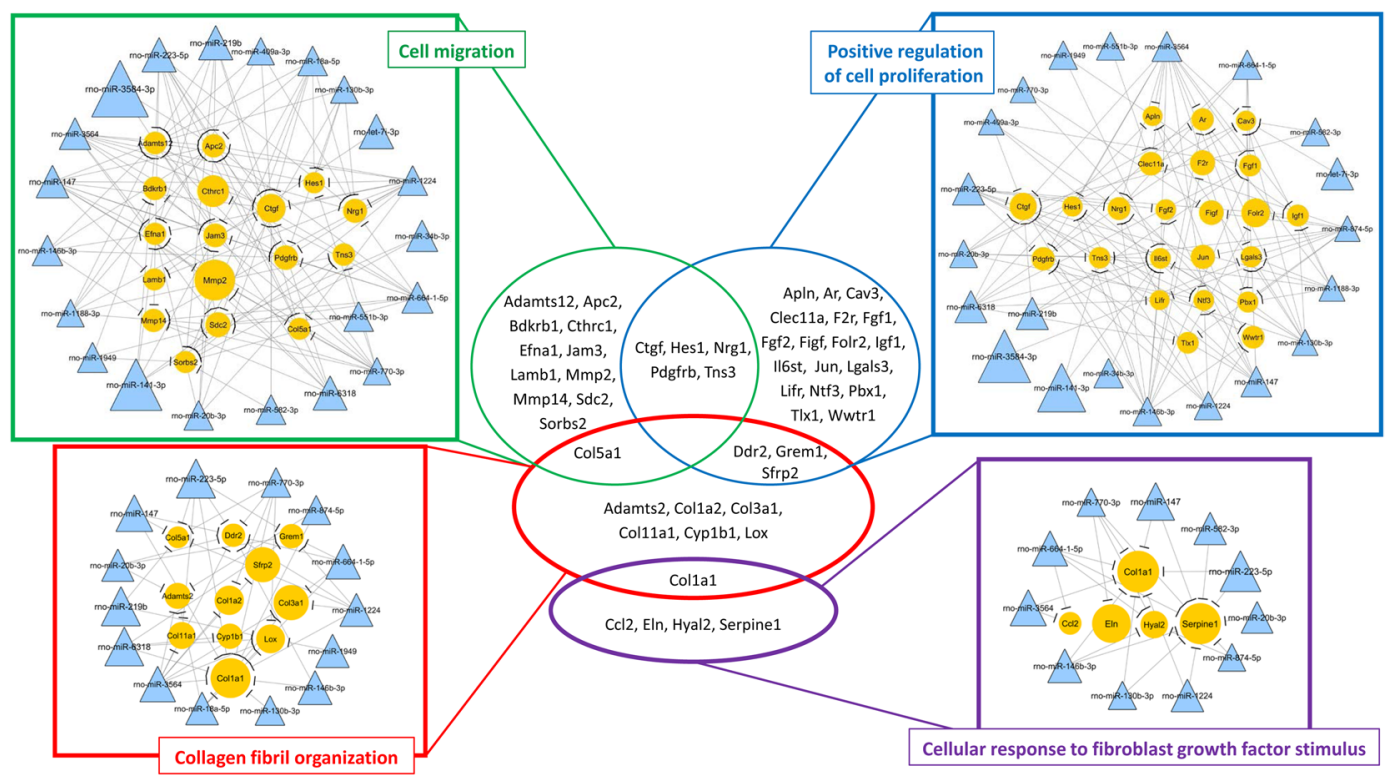

Figure 6. Schematic overview of the specific miRNAs-gene-function relationships identified in the upregulated mRNA and down-regulated miRNA data analyses in the spleens of PPVL rats. The sizes of circles and triangles represent the fold-change in the spleens of PPVL rats compared to those of sham rats.

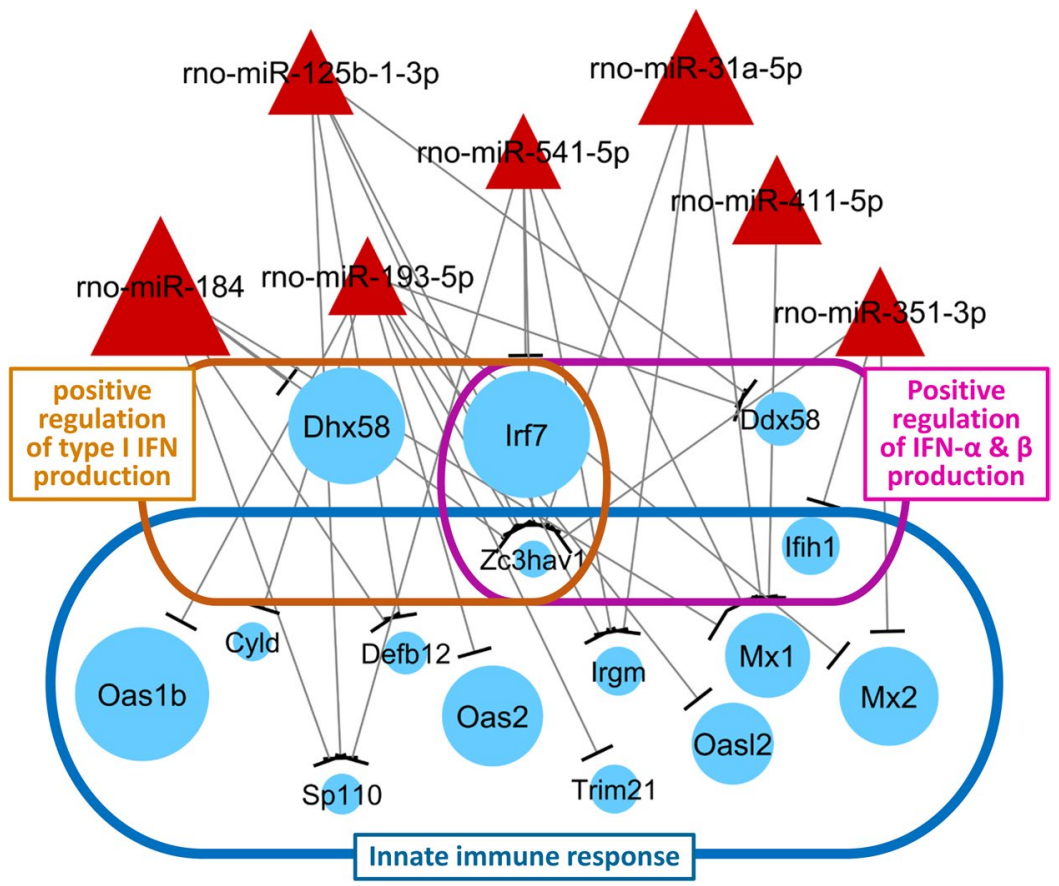

Figure 7. Schematic overview of the specific miRNAs-gene-function relationships identified in the downregulated mRNA and up-regulated miRNA data analyses in the spleens of PPVL rats. The sizes of circles and triangles represent the fold-change in the spleens of PPVL rats compared to those of sham rats.

Microarray data analysis. Differentially expressed transcripts (DETs) were analyzed with Transcriptome Analysis Console 4.0 (Thermo Fisher Scientific) (Fig. 1). The Robust Multiarray Average algorithm was used for normalization of the data to generate a single expression value for each probe set. Normalized expression values were $\log _{2}$-transformed, and empirical Bayes analysis ${ }^{52}$ was performed for differential expression analysis. We identified DETs using the following selection criteria: an empirical Bayes approach with a significance criterion of $\mathrm{p}<0.05$ (for mRNAs) or $<0.10$ (for miRNAs) and a threshold fold change cut off value of $>|1|$ (for mRNAs) or $>|1.5|$ (for miRNAs) in the spleens of PPVL rats compared to those of sham rats. Our purpose was to determine a comprehensive profile of DETs in whole spleen tissues rather than specific cell populations. Therefore, more stringent selection criteria were not used to determine reproducible gene lists ${ }^{53}$ and to avoid limiting the datasets 


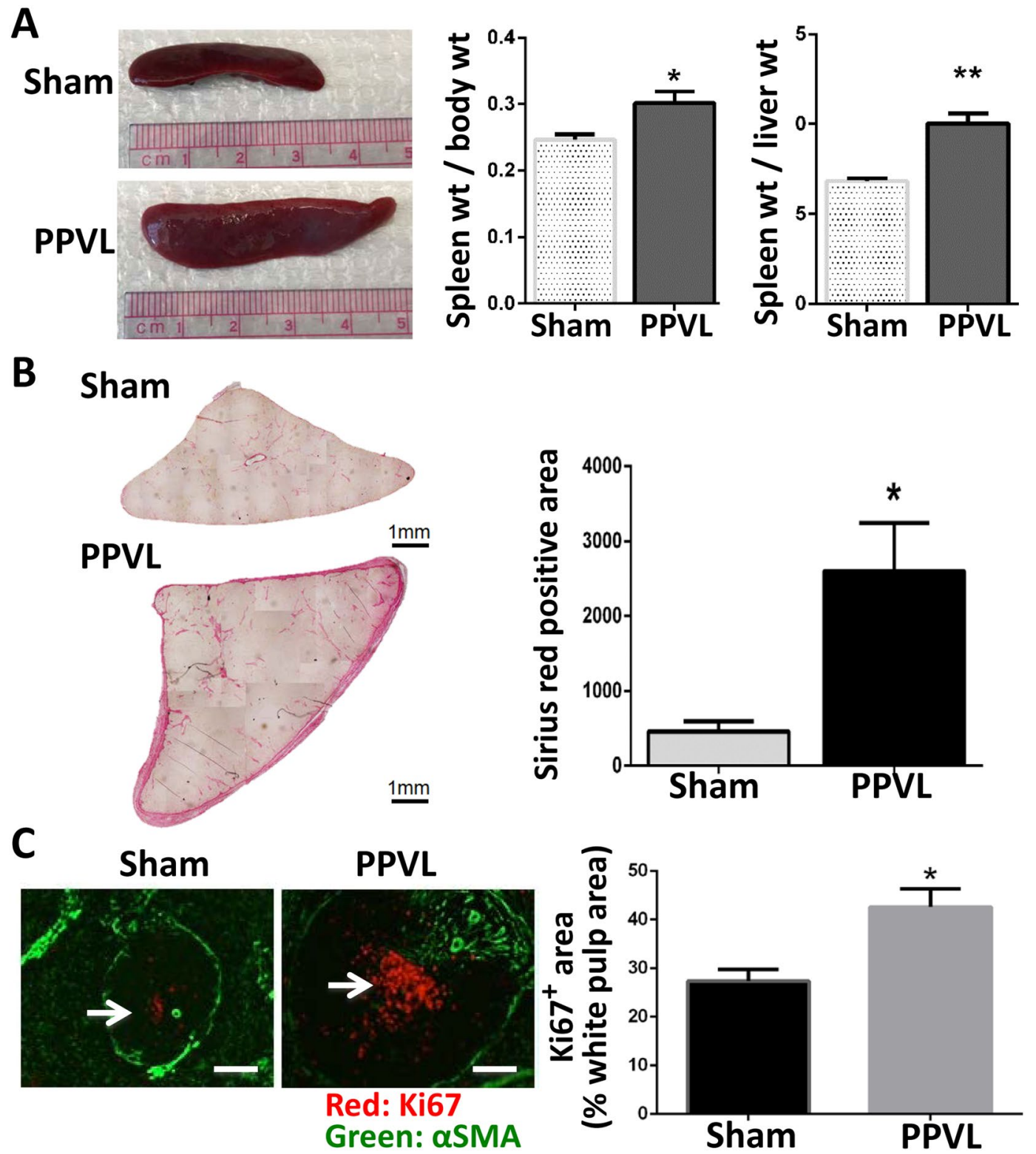

Figure 8. Splenomegaly is associated with increased spleen fibrosis and cell proliferation in rats. (A) Comparison of the spleens between sham and PPVL 10-day rats. “*” and “**” indicate significance at $\mathrm{p}<0.01$ and $\mathrm{p}<0.001$, respectively. (B) Sirius red images of spleens isolated from sham and 10-day PPVL rats. “*” indicates significance at $\mathrm{p}<0.05$. (C) Immunolabeling of Ki67 and alpha-smooth muscle actin $(\alpha-S M A)$. Ki67 was used as a marker for proliferation. Scale bar: $200 \mu \mathrm{m}$. Arrows: Ki67 positive cells in the white pulp area. “*” indicates significance at $\mathrm{p}<0.01$.

unnecessarily ${ }^{54}$. We displayed DETs in a scatter plot and heatmap with dendrogram where a distance metric was the Euclidean distance, and the distances between clusters were computed using the complete linkage method to profile gene expression patterns in the spleens of PPVL rats compared to those of sham rats.

Functional annotation. We first converted all Affymetrix transcript cluster IDs of mRNAs to the corresponding Entrez gene IDs using DAVID version 6.8 (https://david.ncifcrf.gov/home.jsp) ${ }^{55}$ (Fig. 1) with the use of Rattus norvegicus genome as the background list for over-representation analysis. Next, we performed enriched GO analysis using the DAVID ${ }^{55}$ (Fig. 1) to categorize identified genes (i.e., DETs) into biological functions (specific ontology 'terms') constructed by biological process, molecular function and cellular component ${ }^{15,16}$. Significantly enriched biological processes with Benjamini's corrected p-values of $<0.05$ were determined using the DAVID Functional Annotation Tool. The fold enrichment was also calculated by the ratio of the number of genes in the gene set to the expected number in the category based on the human database ${ }^{55}$. We also used the Cytoscape software (http://www.cytoscape.org/index.html) to visualize the results of GO analyses (Fig. 3). To determine main biological pathways in which up- or down-regulated genes are involved, pathway 
analysis was performed using a major public pathway-related database, KEGG (http://www.genome.jp/kegg/) (Fig. 1). In this pathway analysis, we looked into three areas of the KEGG Pathway Database, "Cellular Processes", "Environmental Information Processing" and "Organismal Systems", which are closely related to physiological and pathophysiological processes, and determined specific pathways that contain more than five genes up- or down-regulated in the spleens of PPVL rats.

miRNA target prediction. Target genes for differentially expressed miRNAs were identified using miRGate (http://mirgate.bioinfo.cnio.es/miRGate/) that is composed of all gene information from EnsEMBL (http:// useast.ensembl.org/index.html) and all miRNA data from miRbase (http://www.mirbase.org/) and that processes data using 5 computational tools (miRanda, RNAHybrid, Pita, microtar and Targetscan) and 4 validation tools (Mirtarbase, Mirecords, Tarbase and OncomiRDB) ${ }^{56}$. We used the miRGate database because it contains the most substantial number of rat miRNA sequences for prediction with a high degree of accuracy ${ }^{56}$. For target gene prediction, we focused on miRNAs that were differentially expressed in the spleens of PPVL rats with a fold-change of 1.5 or more. We extracted the genes which were up- and down-regulated in the spleens of PPVL rats from target genes predicted by miRGate of down- and up-regulated miRNAs, respectively. This process also revealed how much genes identified in PPVL spleens were identical with predicted target genes. Similarly, we looked into genes related to the GO terms and KEGG pathways determined as described above and verified that they were also largely predicted traget genes (Fig. 1).

\section{Data Availability}

The datasets generated and/or analyzed in this study are available in the National Center for Biotechnology Information Gene Expression Omnibus repository with unique persistent identifiers of NCBI tracking system accession numbers: GSE113613 and GSE113612, and hyperlinks to the datasets are provided below. https://www. ncbi.nlm.nih.gov/geo/query/acc.cgi?acc=GSE113613. https://www.ncbi.nlm.nih.gov/geo/query/acc.cgi?ac$\mathrm{c}=\mathrm{GSE} 113612$.

\section{References}

1. Mebius, R. E. \& Kraal, G. Structure and function of the spleen. Nat Rev Immunol 5, 606-616 (2005).

2. Bronte, V. \& Pittet, M. J. The spleen in local and systemic regulation of immunity. Immunity 39, 806-818 (2013).

3. Udell, J. A. et al. Does this patient with liver disease have cirrhosis? JAMA 307, 832-842 (2012).

4. Garcia-Tsao, G., Abraldes, J. G., Berzigotti, A. \& Bosch, J. Portal hypertensive bleeding in cirrhosis: Risk stratification, diagnosis, and management: 2016 practice guidance by the American Association for the study of liver diseases. Hepatology 65, 310-335 (2017).

5. Colecchia, A. et al. A combined model based on spleen stiffness measurement and Baveno VI criteria to rule out high-risk varices in advanced chronic liver disease. J Hepatol 69, 308-317 (2018).

6. Kawanaka, H. et al. Effect of laparoscopic splenectomy on portal haemodynamics in patients with liver cirrhosis and portal hypertension. Br J Surg 101, 1585-1593 (2014).

7. Iwakiri, Y. Pathophysiology of portal hypertension. Clin Liver Dis 18, 281-291 (2014).

8. Li, L. et al. The spleen in liver cirrhosis: revisiting an old enemy with novel targets. J Transl Med 15, 111, https://doi.org/10.1186/ s12967-017-1214-8 (2017).

9. Bartel, D. P. MicroRNAs: genomics, biogenesis, mechanism, and function. Cell 116, 281-297 (2004).

10. O'Connell, R. M., Rao, D. S. \& Baltimore, D. microRNA regulation of inflammatory responses. Annu Rev Immunol 30, 295-312 (2012).

11. O’Neill, L. A., Sheedy, F. J. \& McCoy, C. E. MicroRNAs: the fine-tuners of Toll-like receptor signalling. Nat Rev Immunol 11, 163-175 (2011).

12. Li, Y. \& Shi, X. MicroRNAs in the regulation of TLR and RIG-I pathways. Cell Mol Immunol 10, 65-71 (2013).

13. Bosch, J. \& Iwakiri, Y. The portal hypertension syndrome: etiology, classification, relevance, and animal models. Hepatol Int 12, 1-10 (2018).

14. Maglott, D., Ostell, J., Pruitt, K. D. \& Tatusova, T. Entrez Gene: gene-centered information at NCBI. Nucleic Acids Res 39, D52-57 (2011).

15. Ashburner, M. et al. Gene ontology: tool for the unification of biology. The Gene Ontology Consortium. Nat Genet 25, 25-29 (2000).

16. The Gene Ontology Consortium. Expansion of the Gene Ontology knowledgebase and resources. Nucleic Acids Res 45, D331-D338 (2017).

17. Loo, Y. M. \& Gale, M. Jr. Immune signaling by RIG-I-like receptors. Immunity 34, 680-692 (2011).

18. Li, X. Y. et al. Triptolide Restores Autophagy to Alleviate Diabetic Renal Fibrosis through the miR-141-3p/PTEN/Akt/mTOR Pathway. Mol Ther Nucleic Acids 9, 48-56 (2017).

19. Liu, C. et al. MicroRNA-141 suppresses prostate cancer stem cells and metastasis by targeting a cohort of pro-metastasis genes. Nat Commun 8, 14270, https://doi.org/10.1038/ncomms14270 (2017).

20. Feng, J., Xue, S., Pang, Q., Rang, Z. \& Cui, F. miR-141-3p inhibits fibroblast proliferation and migration by targeting GAB1 in keloids. Biochem Biophys Res Commun 490, 302-308 (2017).

21. Fan, G. et al. Anti-Inflammatory Activity of Tanshinone IIA in LPS-Stimulated RAW264.7 Macrophages via miRNAs and TLR4-NFkappaB Pathway. Inflammation 39, 375-384 (2016).

22. Lee, H. M., Kim, T. S. \& Jo, E. K. MiR-146 and miR-125 in the regulation of innate immunity and inflammation. $B M B$ Rep 49 , 311-318 (2016)

23. Lehrer, R. I. \& Lu, W. alpha-Defensins in human innate immunity. Immunol Rev 245, 84-112 (2012).

24. Holly, M. K., Diaz, K. \& Smith, J. G. Defensins in Viral Infection and Pathogenesis. Annu Rev Virol 4, 369-391 (2017)

25. Plager, D. A., Adolphson, C. R. \& Gleich, G. J. A novel human homolog of eosinophil major basic protein. Immunol Rev 179, 192-202 (2001).

26. Ibusuki, R. et al. Transgenic expression of human neutrophil peptide-1 enhances hepatic fibrosis in mice fed a choline-deficient, L-amino acid-defined diet. Liver Int 33, 1549-1556 (2013).

27. Ibusuki, R. et al. Human neutrophil peptide-1 promotes alcohol-induced hepatic fibrosis and hepatocyte apoptosis. PLoS One 12, e0174913 (2017).

28. Han, W., Wang, W., Mohammed, K. A. \& Su, Y. Alpha-defensins increase lung fibroblast proliferation and collagen synthesis via the beta-catenin signaling pathway. FEBS J 276, 6603-6614 (2009).

29. Pearce, E. J. \& MacDonald, A. S. The immunobiology of schistosomiasis. Nat Rev Immunol 2, 499-511 (2002).

30. Burke, M. L. et al. Co-ordinated gene expression in the liver and spleen during Schistosoma japonicum infection regulates cell migration. PLoS Negl Trop Dis 4, e686, https://doi.org/10.1371/journal.pntd.0000686 (2010). 
31. Mejias, M. et al. Relevance of the mTOR signaling pathway in the pathophysiology of splenomegaly in rats with chronic portal hypertension. J Hepatol 52, 529-539 (2010).

32. Chen, Y. et al. Rapamycin Attenuates Splenomegaly in both Intrahepatic and Prehepatic Portal Hypertensive Rats by Blocking mTOR Signaling Pathway. PLoS One 11, e0141159, https://doi.org/10.1371/journal.pone.0141159 (2016)

33. Wozniak, M. A. \& Chen, C. S. Mechanotransduction in development: a growing role for contractility. Nat Rev Mol Cell Biol 10, 34-43 (2009).

34. Dupont, S. Role of YAP/TAZ in cell-matrix adhesion-mediated signalling and mechanotransduction. Exp Cell Res 343, 42-53 (2016).

35. Lipson, K. E., Wong, C., Teng, Y. \& Spong, S. CTGF is a central mediator of tissue remodeling and fibrosis and its inhibition can reverse the process of fibrosis. Fibrogenesis Tissue Repair 5, S24, https://doi.org/10.1186/1755-1536-5-S1-S24 (2012).

36. Ghosh, A. K. \& Vaughan, D. E. PAI-1 in tissue fibrosis. J Cell Physiol 227, 493-507 (2012).

37. Cross, M. J. \& Claesson-Welsh, L. FGF and VEGF function in angiogenesis: signalling pathways, biological responses and therapeutic inhibition. Trends Pharmacol Sci 22, 201-207 (2001).

38. Kotani, K. et al. Comprehensive Screening of Gene Function and Networks by DNA Microarray Analysis in Japanese Patients with Idiopathic Portal Hypertension. Mediators Inflamm 2015, 349215, https://doi.org/10.1155/2015/349215 (2015).

39. Li, Z. et al. MicroRNAome of splenic macrophages in hypersplenism due to portal hypertension in hepatitis B virus-related cirrhosis. Exp Biol Med (Maywood) 233, 1454-1461 (2008).

40. Jiang, A. et al. miR-615-3p promotes the phagocytic capacity of splenic macrophages by targeting ligand-dependent nuclear receptor corepressor in cirrhosis-related portal hypertension. Exp Biol Med (Maywood) 236, 672-680 (2011).

41. Kang, N. \& Koo, J. Olfactory receptors in non-chemosensory tissues. BMB Rep 45, 612-622 (2012).

42. Walensky, L. D. et al. Two novel odorant receptor families expressed in spermatids undergo 5'-splicing. J Biol Chem 273, 9378-9387 (1998).

43. Blache, P., Gros, L., Salazar, G. \& Bataille, D. Cloning and tissue distribution of a new rat olfactory receptor-like (OL2). Biochem Biophys Res Commun 242, 669-672 (1998).

44. Chen, X., Xie, D., Zhao, Q. \& You, Z. H. MicroRNAs and complex diseases: from experimental results to computational models. Brief Bioinform, Epub ahead of print. https://doi.org/10.1093/bib/bbx130 (2017).

45. Chen, X., Wang, L., Qu, J., Guan, N. N. \& Li, J. Q. Predicting miRNA-disease association based on inductive matrix completion. Bioinformatics, Epub ahead of print; 10.1093/bioinformatics/bty503 (2018).

46. Chen, X. et al. BNPMDA: Bipartite Network Projection for MiRNA-Disease Association prediction. Bioinformatics 34, 3178-3186 (2018).

47. Chen, X., Yin, J., Qu, J. \& Huang, L. MDHGI: Matrix Decomposition and Heterogeneous Graph Inference for miRNA-disease association prediction. PLoS Comput Biol 14, e1006418, https://doi.org/10.1371/journal.pcbi.1006418 (2018).

48. Chen, X. \& Huang, L. LRSSLMDA: Laplacian Regularized Sparse Subspace Learning for MiRNA-Disease Association prediction. PLoS Comput Biol 13, e1005912, https://doi.org/10.1371/journal.pcbi.1005912 (2017).

49. Kwon, S. Y., Groszmann, R. J. \& Iwakiri, Y. Increased neuronal nitric oxide synthase interaction with soluble guanylate cyclase contributes to the splanchnic arterial vasodilation in portal hypertensive rats. Hepatol Res 37, 58-67 (2007).

50. Park, J. K. et al. An endoplasmic reticulum protein, Nogo-B, facilitates alcoholic liver disease through regulation of kupffer cell polarization. Hepatology 65, 1720-1734 (2017).

51. Schmitz, J. C. et al. Pigment epithelium-derived factor regulates early pancreatic fibrotic responses and suppresses the profibrotic cytokine thrombospondin-1. Am J Pathol 179, 2990-2999 (2011).

52. Datta, S. \& Datta, S. Empirical Bayes screening of many p-values with applications to microarray studies. Bioinformatics 21, 1987-1994 (2005).

53. MACQ Consortium. et al. The MicroArray Quality Control (MAQC) project shows inter- and intraplatform reproducibility of gene expression measurements. Nat Biotechnol 24, 1151-1161 (2006).

54. Dalman, M. R., Deeter, A., Nimishakavi, G. \& Duan, Z. H. Fold change and p-value cutoffs significantly alter microarray interpretations. BMC Bioinformatics 13(Suppl 2), S11, https://doi.org/10.1186/1471-2105-13-S2-S11 (2012).

55. Huang da, W., Sherman, B. T. \& Lempicki, R. A. Systematic and integrative analysis of large gene lists using DAVID bioinformatics resources. Nat Protoc 4, 44-57 (2009).

56. Andres-Leon, E., Gonzalez Pena, D., Gomez-Lopez, G. \& Pisano, D. G. miRGate: a curated database of human, mouse and rat miRNA-mRNA targets. Database (Oxford) 2015, bav035, https://doi.org/10.1093/database/bav035 (2015).

\section{Acknowledgements}

This study was supported by NIH grants (R01DK116540, R01AA025342, R21AA023599) to YI, JPSP Program for Advancing Strategic International Networks to Accelerate the Circulation of Talented Researchers (No. S2803) to JS, postgraduate fellowship from the Chinese Scholarship Council to CD, and NIH grant (T32 007356) to MM. We would like to thank Dr. Guilin Wang (Yale Center for Genome Analysis) for the guidance of mRNA preparation and running the Affymetrix Gene Chip Array and Yuki Sakamoto for assistance with data management of bioinformatic analyses.

\section{Author Contributions}

Y.I. designed and supervised the study, performed spleen sampling, interpreted obtained data, and wrote the manuscript. J.S. performed statistical analysis of microarray, functional analysis, statistical and gene network analysis, and wrote an initial draft of the manuscript. C.D. isolated RNA, obtained microarray data, and performed histological assessments. M.T. critically reviewed the results from statistical analysis of microarray, functional analysis, statistical and gene network analysis. M.M. critically reviewed and wrote the manuscript. T.U. performed PPVL surgery and collected spleen samples, contributed to the design of the study, and critically revised the manuscript. All authors read and approved the final manuscript.

\section{Additional Information}

Supplementary information accompanies this paper at https://doi.org/10.1038/s41598-018-36297-0.

Competing Interests: The authors declare no competing interests.

Publisher's note: Springer Nature remains neutral with regard to jurisdictional claims in published maps and institutional affiliations. 
(i) Open Access This article is licensed under a Creative Commons Attribution 4.0 International License, which permits use, sharing, adaptation, distribution and reproduction in any medium or format, as long as you give appropriate credit to the original author(s) and the source, provide a link to the Creative Commons license, and indicate if changes were made. The images or other third party material in this article are included in the article's Creative Commons license, unless indicated otherwise in a credit line to the material. If material is not included in the article's Creative Commons license and your intended use is not permitted by statutory regulation or exceeds the permitted use, you will need to obtain permission directly from the copyright holder. To view a copy of this license, visit http://creativecommons.org/licenses/by/4.0/.

(C) The Author(s) 2018 